\title{
AEROSOL OPTICAL PROPERTIES ABOVE OPAQUE WATER CLOUDS DERIVED FROM THE CALIOP VERSION 4 LEVEL 1 DATA
}

\author{
Zhaoyan Liu ${ }^{1,2 *}$, David Winker ${ }^{2}$, Ali Omar ${ }^{2}$, Mark Vaughan ${ }^{2}$, Jayanta Kar ${ }^{1,2}$, Charles Trepte ${ }^{2}$, \\ Yongxiang $\mathrm{Hu}^{2}$, Gregory Schuster ${ }^{2}$, and Stuart Young ${ }^{3}$ \\ ${ }^{1}$ Science Systems and Applications Inc. Hampton, VA23666, USA, *Email: Zhaoyan.liu@nasa.gov \\ ${ }^{2}$ NASA Langley Research Center, Hampton, VA23681, USA \\ ${ }^{3}$ CSIRO Oceans and Atmosphere, Aspendale, Victoria, 3195, Australia
}

\begin{abstract}
In a previous study we evaluated the above-cloud aerosol optical depth (AOD) retrieval at $532 \mathrm{~nm}$ in the CALIOP version 3 (V3) data products for two selected spatial domains along the Saharan dust transport pathway and African smoke transport pathway. In that study we rescaled the V3 level-1 (L1) data to compensate for known V3 calibration biases, and then derived aerosol intrinsic properties such as lidar ratio $\left(S_{a}\right)$ and particulate depolarization ratio (PDR) for comparison with the CALIOP dust and smoke aerosol models. The calibration of the recently released version 4 (V4) CALIPSO L1 data product is significantly improved over V3. So in this paper we repeat our previous analysis using the new V4 L1 data. A comparison shows that our rescaled V3 and the new V4 data are different only by $\sim 1 \%$ in the two selected spatial domains. The retrieved AOD values decrease by $\sim 2.6 \%$ in both domains from $\mathrm{V} 3$ to V4. When the data is screened to exclude weakly scattering layers, the median $S_{a}$ retrieved from the V4 L1 data in the dust transport region is reduced by $1.4 \mathrm{sr}$ to $43.0 \pm 8.3 \mathrm{sr}$. The median $S_{\mathrm{a}}$ value in the smoke transport region is increased by $0.8 \mathrm{sr}$ to $71.2 \pm 15.1$ sr. The PDR values remain almost unchanged for the screened data.
\end{abstract}

\section{INTRODUCTION}

Beginning in June 2006, a global data set of heightresolved measurements of aerosols and clouds has been continuously acquired by the Cloud-Aerosol Lidar with Orthogonal Polarization (CALIOP), deployed aboard the Cloud-Aerosol Lidar and Infrared Pathfinder Satellite Observations (CALIPSO) platform. These active sensor data offer a new and unique opportunity to characterize the global three-dimensional (3-D) distribution of aerosols [1]. Aerosol extinction profiles and AOD can be derived from the CALIOP measurements even for aerosols located over clouds or other bright surfaces. CALIOP's ability to quantify the spatial distribution and optical properties of abovecloud aerosols represents an important step forward, as this information is required to more accurately assess aerosol intercontinental transport and radiative and climate impacts.

In a previous study [2] we evaluated the abovecloud aerosol extinction and optical depth retrieval at $532 \mathrm{~nm}$ in the CALIOP V3 data products for two selected spatial domains along the Saharan dust transport pathway and African smoke transport pathway (refer to Fig. 1). In that study, we also derived the aerosol intrinsic properties such as $S_{a}$ and PDR and compared them with the CALIOP dust and smoke aerosol models. Six years of the V3 nighttime data were analyzed. However, in the V3 data release, stratospheric aerosols in the calibration region (30-34 km) introduced a low bias of a few percent into the L1 532-nm attenuated backscatter profiles [3]. For this reason, all the L1 profiles were recalibrated using a scaling factor determined by comparing the backscatter signals from a higher altitude range $(36-40 \mathrm{~km})$ to the reference molecular scattering signal [4].

The CALIPSO project recently released a new version (V4) of L1 data in which the calibrations have been improved significantly. Major changes at $532 \mathrm{~nm}$ include moving the molecular normalization region up to $36-39 \mathrm{~km}$ (from 30-34 $\mathrm{km}$ in V3), thus greatly reducing calibration biases due to stratospheric aerosols. In this paper, we repeat our earlier analyses using the new V4 L1 data in order to further evaluate the dust and smoke aerosol intrinsic properties reported in our previous study.

\section{METHODOLOGY}

$\mathrm{Hu}$ et al. [5] developed a technique that uses CALIOP measurements of opaque water clouds (OWCs) as a reference to determine the optical depth of overlying transparent aerosol or cirrus layers. This approach takes advantage of the well- 
behaved relationship between the layer-integrated depolarization ratio and the multiple scattering in water clouds. The optical depth for aerosol layers above an opaque water cloud can be derived using

$$
\tau_{\text {aerosol }}=-\frac{1}{2} \ln \left(\frac{\mathrm{H} \gamma_{W C, T S}^{\prime}}{\gamma_{W C, S S, N A}^{\prime}}\right),
$$

where $\gamma_{W C, T S}^{\prime}=\int_{r_{W C, \text { top }}}^{r_{W C, \text { base }}} B^{\prime}(r) d r$ is the integrated attenuated backscatter within the underlying OWC, $B^{\prime}$ is the attenuated backscatter corrected for the attenuation due to the molecular scattering and ozone absorption, and $\mathrm{H}$ is the $\mathrm{OWC}$ layer effective multiple scattering factor [5] defined as

$$
\mathrm{H}=\left(\frac{1-\delta_{I}}{1+\delta_{I}}\right)^{2} .
$$

In equations (1) and (2), $\delta_{I}$ is the layer-integrated volume depolarization ratio within the OWC and $B^{\prime}$ is the attenuated backscatter coefficient corrected for molecular and ozone attenuation, and the subscripts WC, SS and TS represent, respectively, water clouds, single scattering and total scattering (i.e., single scattering and multiple scattering together).

The denominator in Eq. (1), $\gamma_{W C, S S, N A}^{\prime}$, is obtained from a climatology of the single-scattering integrated attenuated backscatter for OWCs that have no aerosol (NA) or any other features detected above. A location dependent climatology of $\gamma_{W C, S S, N A}^{\prime}$ was developed from six years (20072012) of the CALIOP data to account for the spatial variability in the microphysical properties of water clouds [2].

Retrievals using the OWC technique are performed on CALIOP L1 attenuated backscatter profiles, averaged horizontally to $5 \mathrm{~km}$. The OWCs selected for constrained retrievals are (1) single layered with (2) top heights less than $2 \mathrm{~km}$ for which (3) opaque water clouds are detected in all 15 singleshot profiles within each $5-\mathrm{km}$ average, and the standard deviation of these 15 single shot top heights is less than $50 \mathrm{~m}$. More details can be found in Liu et al. [2].

The AOD derived using the OWC technique can subsequently be used as a constraint to retrieve $S_{a}$, PDR, extinction and backscatter coefficients for the overlying aerosol layer using a two-component solution of the lidar equation [6].

\section{RESULTS}

Six years (2007-2012) of the CALIOP V4 L1 data have been analyzed. To select OWCs, the V3 L2 vertical feature mask (VFM) data are used, because the V4 L2 data products have not yet been created. As in our previous study, we focus our analysis in this paper on the two selected regions shown in Fig. 1. North Africa is the largest source of dust emissions in the world, and transport of Saharan dust across the tropical North Atlantic reaches a maximum during the summer. For this reason, the months of June - August are selected for analyzing the dust transport region. Outbreaks of smoke due to biomass burning in southern Africa are most frequent in the months of July - September, and we therefore select these months for analyzing the smoke transport region.

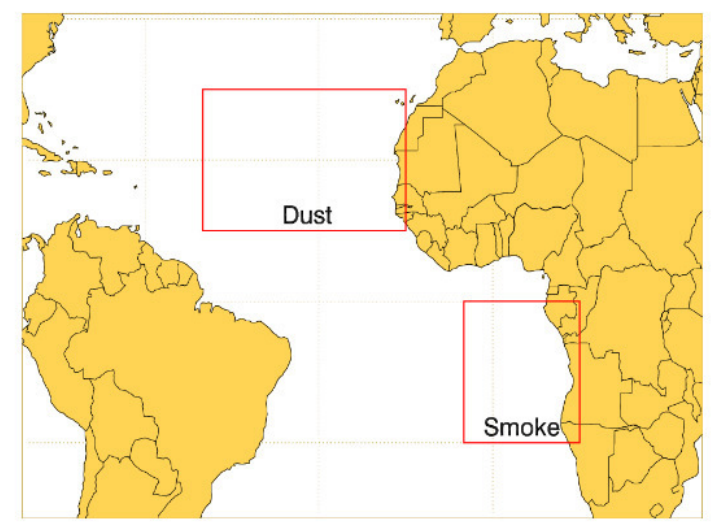

Figure 1 Spatial domains analyzed (red boxes). The northern region $\left(10^{\circ} \mathrm{N}-30^{\circ} \mathrm{N}, 50^{\circ} \mathrm{W}-15^{\circ} \mathrm{W}\right)$ is along the Saharan dust transport pathway over the tropical North Atlantic, while the southern region $\left(20^{\circ} \mathrm{S}-0^{\circ}, 5^{\circ} \mathrm{W}\right.$ $15^{\circ} \mathrm{E}$ ) is along the smoke transport pathway over the tropical South Atlantic.

Figures 2 and 3 present results from the spatial domains indicated by the red boxes in Fig. 1. The mean value of the dust $S_{a}$ distribution is $47.1 \mathrm{sr}$, with a median of $43.5 \mathrm{sr}$, a mode of $42.5 \mathrm{sr}$, and a standard deviation of $24.5 \mathrm{sr}$. For the PDR distribution the mean is 0.255 , the median is 0.275 , the mode is 0.279 , and the standard deviation is 1.64 . For the screened data using attenuated scattering ratio $\left(\mathrm{ASR}=\int_{W C, \text { top }}^{8 k m} B^{\prime} d r / \int_{W C, \text { top }}^{8 k m} \beta_{m} d r-1\right)>0.3$, the mean/median/mode values are 43.4/43.0/42.5 \pm 8.3 for $S_{a}$, and $0.280 / 0.281 / 0.282 \pm 0.045$ for PDR. 

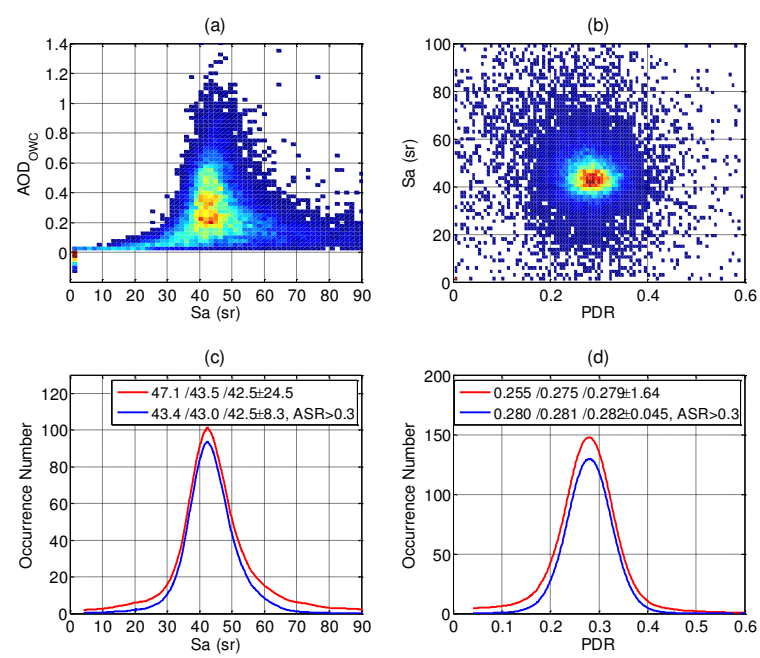

Figure 2 Analysis results for the dust transport region as shown in Fig. 1. The upper row shows 2-D distributions of (a) $A_{O D}$ owc vs. $S_{a}$ retrieved using $A_{O D D}$ as a constraint and (b) $S_{a}$ vs. PDR, while the lower row shows histograms of (c) $\mathrm{S}_{\mathrm{a}}$ and (d) PDR occurrence frequencies. The red curves in (c) and (d) include all data and the blue curves are screened data using ASR $>0.3$. The numbers in the legends of (c) and (d) show mean/median/mode \pm standard deviation.
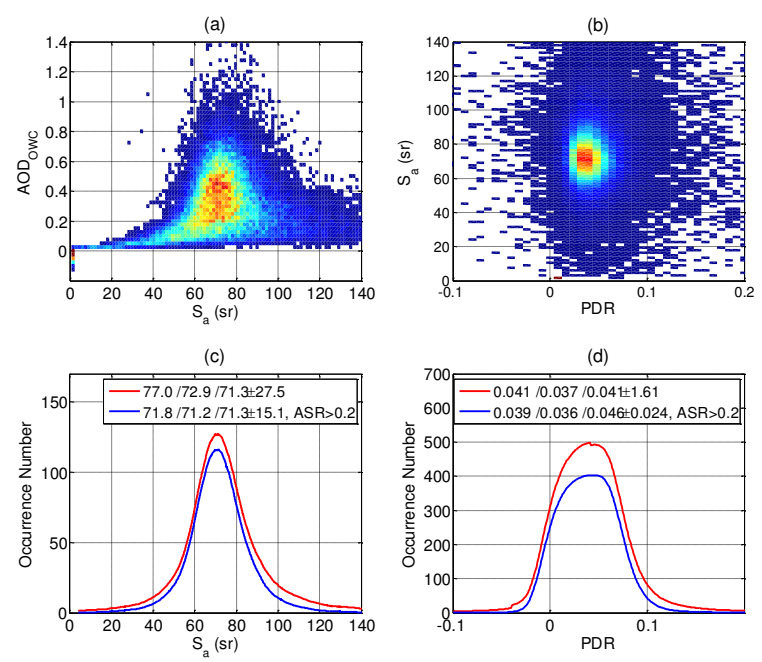

Figure 3 Analysis results for the smoke transport region as shown in Fig. 1. The upper row shows 2-D distributions of (a) $\mathrm{AOD}_{\mathrm{OWC}}$ vs. $\mathrm{S}_{\mathrm{a}}$ retrieved using

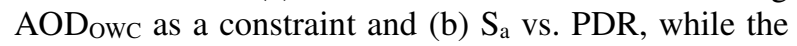
lower row shows histograms of (c) $S_{a}$ and (d) PDR occurrence frequencies. The red curves in (c) and (d) include all data and the blue curves are screened data using ASR $>0.2$. The numbers in the legends of (c) and (d) show mean $/$ median $/$ mode \pm standard deviation.

The $S_{a}$ values retrieved in the smoke transport region have mean/median/mode values of 77.0 / $72.9 / 71.3 \pm 27.5 \mathrm{sr}$ for all the data and 71.8/71.2/
$71.3 \pm 15.1 \mathrm{sr}$ for the screened data using ASR $>0.2$. The PDR values retrieved in the smoke region are typically smaller than 0.1 , with mean/median/ mode values of $0.041 / 0.037 / 0.041 \pm 1.61$ for all smoke layers analyzed and 0.039/0.036/0.046 \pm 0.024 for the layers with $\mathrm{ASR}>0.2$.

\section{COMPARISONS}

Figure 4 presents a comparison of the $S_{a}$ and PDR retrieved in the dust and smoke transport regions from the rescaled V3 and standard V4 L1 data using the OWC constrained retrieval. In this comparison, weakly scattering aerosol layers were screened out using the ASR test. In general, the retrieved PDR values from the two L1 datasets have an almost identical distribution, because the PDR retrieval is largely insensitive to calibration errors [7]. Small difference is seen in the median retrieved $S_{a}$ value in the dust transport region (decreased by $1.4 \mathrm{sr}$, or $3.2 \%$ ), while the $\mathrm{S}_{\mathrm{a}}$ difference for the smoke transport region is even smaller (increased by $0.8 \mathrm{sr}$, or $1.1 \%$ ).
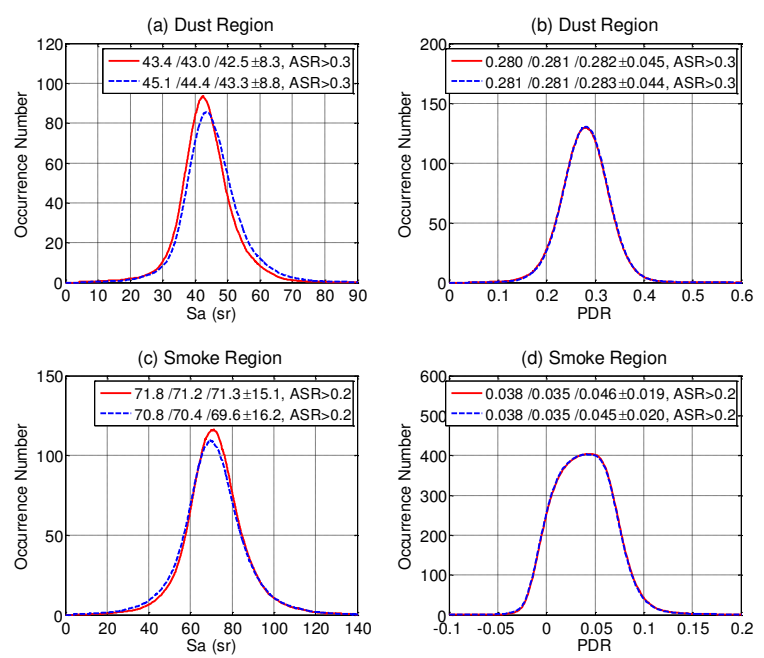

Figure $4 \mathrm{~S}_{\mathrm{a}}$ and PDR retrieved using the OWC constrained technique in the dust ( $\mathrm{a}$ and $\mathrm{b}$ ) and smoke ( $\mathrm{c}$ and d) transport regions from the rescaled V3 (blue) and V4 (red) L1 data, with weakly scattering aerosol layers screened out.

We examine the calibration in the two L1 datasets. As shown in Fig. 5, the rescaled V3 L1 data is, on average, $0.8 \%$ smaller than the V4 L1 data in the dust transport region and $1.1 \%$ larger in the smoke transport region. These differences are small and within the estimated calibration uncertainties [3]. On the other hand, the retrieved AOD from the V4 data is smaller than the V3 data by $0.006(2.6 \%)$ in 
the dust transport region and by $0.08(2.6 \%)$ in the smoke transport region.
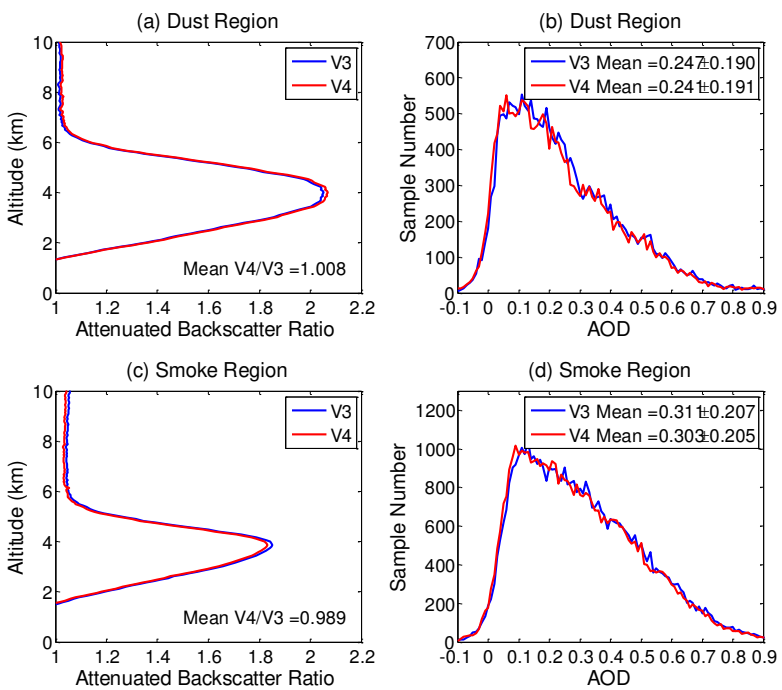

Figure 5 Average attenuated backscatter ratio (a and c) and histograms of AOD (b and d) retrieved from the V3 (blue) and V4 (red) L1 data using the OWC technique.

\section{CONCLUSIONS}

Following on our previous study that analyzed six years of the CALIOP V3 data to retrieve the aerosol optical depth above opaque water clouds and dust and smoke lidar ratio, in this paper we repeated the same analysis using the recently released V4 L1 data. The rescaled V3 L1 data that was used in the previous study is, on average, different from the V4 L1 data by $\sim 1 \%$ in the two selected spatial domains. The retrieved abovecloud aerosol optical depths are $2.6 \%$ smaller using the V4 L1 data than the V3 L1 data in both domains, while the particular depolarization ratio values using both versions have an almost identical distribution for moderately scattering layers because the retrieval of this parameter is largely insensitive to the data calibration. Lidar ratio is an intrinsic aerosol property and a key parameter that limits the accuracy of the extinction retrieval. The retrieved median lidar ratio value is decreased by 1.4 sr using the V4 L1 data in the dust transport region. This is mainly because the V4 L1 data in this region is $\sim 1 \%$ larger than the rescaled V3 L1 data while the above-cloud aerosol optical depths retrieved from the V4 data are $\sim 2.6 \%$ smaller than those retrieved from the rescaled V3 data. In the smoke transport region the lidar ratio is increased by $0.8 \mathrm{sr}$. Unlike in the dust transport region, in the smoke transport region the attenuated backscatter and the retrieved aerosol optical depths are both slightly smaller in the V4 analysis than in the V3 analysis. Because these differences have mutually compensating effects, the difference in the retrieved smoke lidar ratios from the two data versions is smaller than that in the retrieved dust lidar ratios.

This is an on-going study, and it would be useful to perform the same analysis once again when the V4 L2 data products become available.

\section{REFERENCES}

[1] Winker, D. M., J. L. Tackett, B. J. Getzewich, et al.: The global 3-D distribution of tropospheric aerosols as characterized by CALIOP, Atmos. Chem. Phys., 13, 3345-3361, 2013.

[2] Liu, Z., D. Winker, A. Omar, M. Vaughan, et al.: Evaluation of CALIOP 532-nm aerosol optical depth over opaque water clouds, Atmos. Chem. Phys., 15, 1265-1288, 2015.

[3] Rogers, R. R., C. A. Hostetler, J. W. Hair, et al.: Assessment of the CALIPSO Lidar $532 \mathrm{~nm}$ Attenuated Backscatter Calibration Using the NASA LaRC Airborne High Spectral Resolution Lidar, Atmos. Chem. Phys., 11, 1295-1311, 2011.

[4] Vernier, J. P., J. P. Pommereau, A. Garnier, et al.: The tropical stratospheric aerosol layer from CALIPSO lidar observations, J. Geophys. Res., 114, D00H10, 2009.

[5] Hu, Y., M. Vaughan, Z. Liu, et al.: Retrieving optical depths and lidar ratios for transparent layers above opaque water clouds from CALIPSO lidar measurements, IEEE Geosci. Remote Sens. Lett., 4, 523-526, 2007.

[6] Young, S. A., and M. A. Vaughan: The retrieval of profiles of particulate extinction from Cloud Aerosol Lidar Infrared Pathfinder Satellite Observations (CALIPSO) data: Algorithm description, J. Atmos. Oceanic Technol., 26, 11051119, 2009

[7] Liu, Z., T. D. Fairlie, I. Uno, et al.: Transpacific Transport and Evolution of the Optical Properties of Asian Dust, J. Quant. Spectrosc. Ra., 116, 2433, 2013. 